\title{
DISEÑO TELEVISIVO A TRAVÉS DE LA NEUROCIENCIA
}

\section{Television design through neuroscience}

\section{Verónica Crespo-Pereira y Beatriz Legerén-Lago}

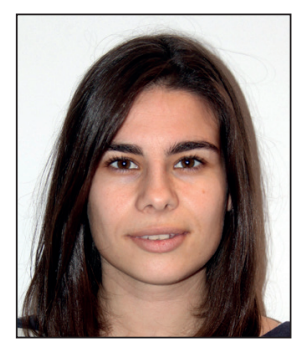

Verónica Crespo-Pereira es licenciada en publicidad y relaciones públicas por la Universidad de Vigo (UVigo) y máster en producción y gestión audiovisual por la Universidad de A Coruña. Ejerce como investigadora y docente en la UVigo donde lleva a cabo su tesis doctoral sobre la implementación de la metodología neurocientífica en el campo de la televisión. Es autora de diversos artículos en revistas de impacto sobre la mencionada temática.

http://orcid.org/0000-0001-7373-7204

Universidad de Vigo, Facultad de Ciencias Sociales y de la Comunicación Campus A Xunqueira, s/n. 36005 Pontevedra, España veronicacrespopereira@gmail.com

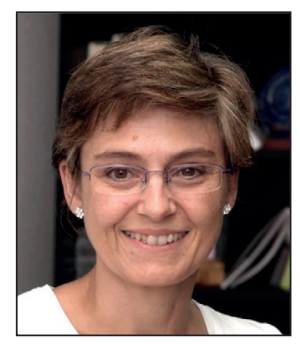

Beatriz Legerén-Lago es investigadora especializada en game studies, además de profesora de la asignatura Videojuegos: Diseño y desarrollo, entre otras materias relacionadas con la creación de proyectos interactivos. Autora de artículos y capítulos de libro sobre diseño de juegos. Ha participado en proyectos de investigación financiados en convocatorias públicas -autonómicas y nacionales- de concurrencia competitiva.

http://orcid.org/0000-0001-6169-0875

Universidad de Vigo, Facultad de Ciencias Sociales y de la Comunicación Campus A Xunqueira, s/n. 36005 Pontevedra, España beatriz.legeren@gmail.com

\section{Resumen}

La neurociencia cognitiva ha permitido comprender mejor el impacto de estímulos mediáticos en el procesamiento cognitivo y emocional. Este artículo aborda una revisión bibliográfica exploratoria sobre la contribución de la neurociencia en el diseño de contenidos audiovisuales ( $\mathrm{N}=57)$. El trabajo se llevó a cabo en tres etapas: a) revisión de información no estructurada; b) análisis, organización y síntesis del contenido; c) conclusiones. Así mismo, entrevistas a expertos en el campo televisivo, consultoría de neuromarketing y neurocientíficos $(\mathrm{N}=10)$ avalan los resultados hallados en la revisión bibliográfica. Como corolario, se aporta un conjunto de pautas fundamentadas científicamente para un diseño eficiente de contenidos televisivos de entretenimiento, educativos e informativos basados en el storytelling, la edición y la creación de nuevas plataformas. La investigación apunta a la emoción y atención como indicadores clave de eficacia para el diseño de productos televisivos.

\section{Palabras clave}

Neurociencia; Neuromarketing; Diseño de contenidos; Televisión; Contenido audiovisual; Contenido televisivo; Emoción; Atención; Narración; Edición; Revisión bibliográfica.

\begin{abstract}
Cognitive neuroscience has provided a better understanding of the impact of media stimuli on cognitive and emotional processing. This article offers an exploratory bibliographic review on the contribution of neuroscience in the design of audiovisual content $(\mathrm{N}=57)$. This review was carried out in three stages: a) review of unstructured information; b) analysis, organization, and synthesis of content; c) conclusions. Likewise, interviews with experts in the television field, neuromarketing consultants, and neuroscientists $(\mathrm{N}=10)$, support the conclusions from the bibliographic review. Subsequently, the paper provides a set of scientifically endorsed guidelines for the efficient design of TV content for entertainment, education, and information, based on storytelling, edition, and the creation of new platforms. The bibliographic research reveals that emotion and attention are the key indicators of effectiveness for the design of television products.
\end{abstract}

\section{Keywords}

Neuroscience; Neuromarketing; Content design; Television; Audiovisual content; Television content; Emotion; Attention; Storytelling; Edition; Literature review; Bibliographic review. 
Crespo-Pereira, Verónica; Legerén-Lago, Beatriz (2017). “Diseño televisivo a través de la neurociencia”. El profesional de la información, v. 26, n. 6, pp. 1047-1055.

\section{Neurociencia y televisión}

El contenido y la estructura narrativa de una producción audiovisual se diseña para atraer la atención y conseguir determinados efectos en la audiencia (Kauttonen; Kaipainen; Tikka, 2014). Desde la aparición del cine, la ciencia ha empleado las películas como vehículo sobre el que realizar estudios empíricos respecto al procesamiento mental y perceptual por su potencial para monitorizar estímulos en tiempo real y simular condiciones reales de visionado con mínimo impacto (Segev; Martínez; Zatorre, 2014).

El visionado televisivo es una actividad mental altamente dinámica, pues requiere de un rápido procesado de grandes cantidades de información visual y auditiva para propiciar el engagement (compromiso) a través de la narrativa y la edición. Aunque el cine se ha investigado con metodologías propias de la psicología del siglo XIX (Shimamura, 2010), la aparición de la neurociencia cognitiva en la década de 1980 y la inclusión de modernas tecnologías consigue los mayores descubrimientos en torno al procesamiento neural ante estímulos audiovisuales (Shimamura, 2013). La neurociencia cognitiva trabaja sobre productos fílmicos y televisivos bajo la asunción de que las películas influyen en las respuestas neurales de los espectadores, y que estados mentales como la emoción están vinculados a estados cerebrales. Además, asume que las respuestas neurales y fisiológicas son fiables y predecibles al conjunto poblacional, toda vez que una misma cinta conseguiría similares respuestas cerebrales (Hasson et al., 2008).

La neurociencia cognitiva tiene potencial para proporcionar pautas de diseño eficiente de contenidos, edición y optimización de nuevas plataformas

La neurociencia cognitiva evidencia la actividad neural y fisiológica de la experiencia audiovisual mediante el empleo de técnicas de registro de actividad neural (IRMf, EEG) o biométricas (eye tracking, heart rate...) (Innerscope Research, 2014). Aunque el impacto fisiológico y neural de los estímulos televisivos ha sido objeto de investigación teórica sobre la percepción visual (Thorson; Lang, 1992), conocer la reacción psicológica y fisiológica del público ante los estímulos del storytelling y la estructura, proporcionan hallazgos relevantes para la industria del entretenimiento y la teoría fílmica (Aldama, 2015). El storytelling se entiende como la forma de contar historias independientemente de la estructura adoptada en cada momento y soporte (Beamish; Beamish, 2015).

Las teorías en torno al procesamiento cognitivo de estímulos estuvieron tempranamente ligadas a la composición fílmica, si bien la demostración empírica no ha tenido lugar hasta la innovación tecnológica de las últimas décadas (Smith, 2013). Además de recurrir a la intuición y a la prueba-error en el diseño de elementos narrativos, visuales y auditivos que atrajesen eficientemente la atención y la emoción del público, cineastas pioneros como Sergei Eisenstein se sintieron atraídos por el estudio de respuestas fisiológicas para diseñar contenidos (Smith, 2013).

La neurociencia contemporánea favorece una mayor comprensión de variables clave para la televisión, como la atención, emoción y memoria (Morin, 2011; Tapia-Frade; Martín-Guerra; Puente, 2016). Su monitorización en relación con elementos estructurales y narrativos, permite determinar grados de efectividad y reglas para diseños eficientes (Steele et al., 2013; Brennan, 2011).

En resumen, un profundo conocimiento sobre el procesamiento de estímulos, ofrece la fundamentación científica adecuada para adaptar contenidos y estructuras televisivas a la fisiología humana (Aldama, 2015). Se ha demostrado que los contenidos audiovisuales contemporáneos han modificado sus pautas estructurales hasta compatibilizarse con patrones de atención y percepción endógenas a nuestra mente (Cutting; DeLong; Brunick, 2011) para conseguir mayor eficiencia en términos de atención y emoción.

\section{Objetivos y metodología}

El presente artículo realiza una revisión bibliográfica sobre la contribución de la neurociencia a la eficiencia del contenido y la estructura televisiva. Los objetivos son:

- analizar las bases teóricas que determinan el potencial de la neurociencia en el desarrollo de productos televisivos eficientes;

- analizar y categorizar las principales investigaciones neurocientíficas sobre diseño televisivo;

- establecer el impacto de la estructura y del contenido televisivo sobre procesamiento cognitivo y emocional;

- extraer pautas para el diseño de contenidos.

La revisión es descriptiva y analítica. Primero se fundamenta el valor de la neurociencia en el estudio de productos televisivos para posteriormente abordar pautas aplicables al diseño de contenidos por categorías de productos que responden a la capacidad de las televisiones de formar, informar y entretener y establecer espacios publicitarios para su sostenimiento.

Se ha realizado una primera consulta exploratoria en Scopus, Web of Science y Google Scholar examinando artículos que aplican metodología neurocientífica ante estímulos televisivos. Las dos primeras bases de datos se seleccionaron por la calidad de sus publicaciones, y la tercera para ampliar el espectro de investigaciones de potencial interés. Las ecuaciones de búsqueda en el campo topic fueron 'neuroscience' AND 'television'; 'neuromarketing' AND 'television'; 'consumer neuroscience' AND 'television'; 'neurociencia te- 
levisión'; 'emotion storytelling brain'. La revisión empleó el método de bola de nieve consistente en acceder a artículos citados en la bibliografía de los documentos iniciales. Una búsqueda complementaria se centró en estudios neurocientíficos financiados por televisiones en la base de datos Warc, dada su relevancia en el diseño de contenidos.

Complementariamente, se cuenta con la opinión de expertos sobre el potencial de la neurociencia en el diseño televisivo a través de entrevistas a profesionales de Grupo Atresmedia y Mediaset España $(\mathrm{N}=2)$, las consultoras de neuromarketing españolas BitBrain, BrainHouse, Sociograph, Neurologyca, Inside Brain ( $\mathrm{N}=5)$ y los neurocientíficos Xurxo Mariño, Luis Martínez-Otero, Susana Martínez-Conde $(\mathrm{N}=3)$. Sus aportaciones fundamentan el análisis de la bibliografía y se incorporan implícitamente en el texto como complemento a la discusión efectuada.

\section{Atención y emoción en el diseño televisivo}

El estudio de la atención en televisión ha sido de interés en los últimos 40 años (Simons et al., 2003), pues se considera necesaria para iniciar el proceso de percepción, cognición y emoción (Hasson et al., 2008; Dudai, 2008). Dos corrientes principales abordan su estudio: el modelo pasivo de consumo mediante el cual la atención es resultado de la fijación de la mirada en la pantalla (Simons et al., 2003; Barreda-Ángeles, 2013) y la corriente propia de la psicología cognitiva que define la atención como la asignación de recursos cognitivos para el procesado de los estímulos y cuyo objeto de estudio es la intensidad atentiva (Thorson, 2011; Barreda-Ángeles, 2013).

El storytelling y la estructura de un producto audiovisual determinan la asignación de recursos y orientan respuestas fisiológicas y conductuales de los espectadores (Lang et al., 2010). Guiar adecuadamente la atención mediante la tensión narrativa (Kauttonen; Kaipainen; Tikka, 2014; Hasson et al., 2008) y la edición produce sincronía atencional, fenómeno que registra patrones de fijación de la mirada compartida por los sujetos (Cohen; Shavalian; Rube, 2015). Las características del producto audiovisual (planos, composición...) determinan el grado de sincronía. El movimiento como elemento para atraer la atención ha sido tratado académicamente. Cuando éste se produce en una pequeña parte de la escena con respecto a una composición estática, generará sincronía (Smith, 2013). El movimiento es un elemento dentro de un complejo sistema. Los planos medios cerrados alcanzan la mayor sincronía atentiva frente otros tipos, y las caras atraen nuestra atención de forma innata (Smith, 2013). Generalmente, la sincronía aumenta en vídeos editados frente al decrecimiento de los no editados (Dorr et al., 2010; Cohen; Shavalian; Rube, 2015). Cuanto más larga una secuencia menor probabilidad de conseguir alta actividad visual frente a secuencias cortas (Cutting; DeLong; Brunick, 2011) pues la familiaridad con el contenido produce un decrecimiento de la atención (Smith, 2013).

La relación emoción-cognición es relevante para la comunicación. La emoción es el motor para conseguir la atención y enganchar al espectador (Aldama, 2015). Resulta científicamente aceptado que la emoción es esencial para la cognición y conducta (Zhou, 2005) siendo clave en la atención: entretenimiento, persuasión (Bolls; Lang; Potter, 2001), memoria (Sharot; Phelps, 2004), razonamiento, toma de decisiones e interpretación de mensajes mediáticos (Zhou, 2005). Aunque todo producto audiovisual busca provocar determinados efectos y emociones, no todas las narrativas impactan igual. La forma en que se procesa la información permite establecer principios para optimizar contenidos (Morin, 2011).

En comunicación, el procesamiento de la emoción es frecuentemente analizado a partir de la teoría dimensional de la emoción (Zhou, 2005). Ésta sugiere que las experiencias emocionales pueden abordarse a partir de dimensiones como la valencia o grado en que una respuesta es positiva o negativa (Bolls; Lang; Potter, 2001), y el arousal o nivel de activación asociado a la respuesta emocional (Jeong; Bohil; Biocca, 2011). El arousal es vital para estudios sobre la eficacia del mensaje al estar vinculado a la atención y el recuerdo (Grabe et al., 2000; Zhou, 2005): a altos niveles de arousal mayor recuerdo (Bolls; Lang; Potter, 2001; Sharot; Phelps, 2004). La teoría ha sido empleada para el estudio de la emoción sobre informativos y mensajes televisivos en general (Zhou, 2005).

La monitorización de variables clave como atención, emoción y engagement (compromiso) en relación con elementos estructurales y narrativos de un producto televisivo, permite determinar grados de efectividad y pautas para el diseño

A continuación se analizan aquellas investigaciones neurocientíficas aplicables al diseño televisivo. Los estudios se categorizaron atendiendo al espectro de contenidos existentes:

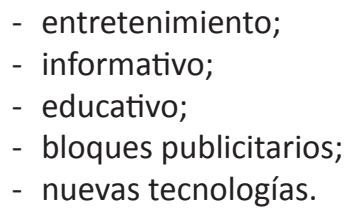

\subsection{Entretenimiento y storytelling}

El ser humano está biológicamente predispuesto al storyteIling (Monarth, 2014) y la información visual. Evolutivamente la visión fue vital para la supervivencia de la especie al desarrollar centros especializados en el cerebro para el procesamiento visual. El cerebro tiene preferencia por atajos mentales en forma de imágenes y experiencias frente a la palabra y largas deliberaciones (Morin, 2011). La metodología neurocientífica permite comprender cómo la estructura y narrativa impacta sobre la atención y emoción (clave en estudios de series de ficción) (Crespo-Pereira; Martínez-Fernández; García-Soidán, 2016; Tapia-Frade; Martín-Guerra, 2016b). Las características del contenido y estructura determinarán la eficacia del mensaje (Brennan, 2011).

La neurobiología del storytelling concluye que la tensión narrativa es necesaria para captar primero la atención y, 
de crear tensión suficiente, empatizar con los personajes, emocionar (Zak, 2014) y conseguir el engagement (Cohen; Shavalian; Rube, 2015). El engagement se emplea tradicionalmente como indicador de desempeño televisivo y se ha analizado de acuerdo con diversas tradiciones metodológicas, constructos y dimensiones (González-Bernal, 2016). Desde la neurociencia, el engagement emocional se ha definido como un constructo subconsciente vinculado a la cantidad de feeling existente durante el procesado de estímulos (Heat, 2007) y es resultado de la ecuación sincronía fisiológica + intensidad (Sawyer, 2014; Siefert et al., 2008).

\section{La emoción y la atención son indicado- res clave de eficacia para el diseño de productos televisivos}

Determinados especialistas manifiestan que el engagement se reduce generalmente tras los tres primeros minutos de un programa y se relaciona con un proceso fisiológico conocido como "habituación" (Seixas et al., 2015). La narrativa es vital para incrementar la atención y modular la emoción. El conflicto y la incertidumbre en toda historia son necesarios para provocar el interés del espectador e incrementar su atención (Tapia-Frade; Martín-Guerra, 2016b). Es necesario un equilibrio. La relación de picos emocionalmente altos y bajos en un producto audiovisual es positiva y necesaria para no causar agotamiento. Por ello los diferentes elementos de un contenido deberán ocupar una posición y una razón de estar adecuada. La música y contenidos visuales impactantes son necesarios para alcanzar picos emocionalmente intensos sin necesidad de que medie la voz (Tapia-Frade; Martín-Guerra, 2016a). Mientras que la lucha de los personajes por lograr sus objetivos atrae al cerebro y genera motivación, persuasión y recuerdo (Zak, 2014), el uso de una estructura narrativa clásica o aristotélica (principio, nudo, desenlace) y cronológica es la que menos esfuerzo requiere a la hora de procesar, resultando más fácil de recordar (Lang; Potter; Grabe, 2003; Mújica; Bachmann, 2016), y ser innatamente más placentera que otras estructuras alternativas (Berliner, 2013). Variaciones sobre esta estructura como la ejemplificada en la Pirámide de Freytag (Monarth, 2014; Welbourne, 2015) emplean la tensión narrativa como elemento para aumentar la atención y emoción del espectador.

\subsection{Contenidos informativos}

La información se ha estudiado en relación con el impacto de la estructura y contenido sobre los niveles de atención y arousal, evidenciando los efectos de la emoción sobre el procesamiento cognitivo. Elementos como efectos de sonido, tono de voz del narrador (Grabe; Zhou; Barnett, 2001), ritmo de presentación de información (Lang; Potter; Grabe, 2003), o la realización (Lang et al., 2010), tienen consecuencias cognitivas y emocionales. Es necesario encontrar un equilibrio entre el contenido y la estructura informativa para un adecuado procesamiento y funcionamiento de la atención y memoria (Lang; Potter; Grabe, 2003).

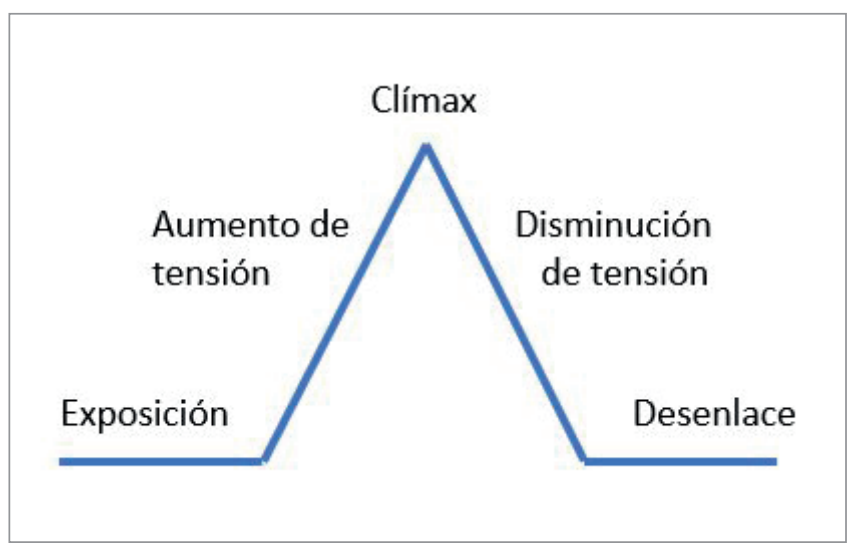

Figura 1. Pirámide de Freytag

Determinados estudios concluyen que los mensajes negativos atrapan más la atención frente a los positivos (Bolls; Lang; Potter, 2001) y que las imágenes negativas consiguen un mayor impacto en la atención (Lang; Newhagen; Reeves, 1996) y arousal frente a imágenes neutras (Simons et al., 2003). Consecuentemente las imágenes negativas más cautivadoras inhiben la identificación (Lang; Newhagen; Reeves, 1996) y recuerdo de la información emplazada antes de las imágenes negativas (Newhagen; Reeves, 1992). Por ello la información importante deberá introducirse siguiendo las escenas negativas y no precediéndolas (Lang; Potter; Grabe, 2003). Aun cuando se trata de contenidos informativos, los elementos y características del storytelling y edición impactan de diversa forma en la emoción y cognición, por ejemplo, con los informativos de estilo melodramático (Grabe et al., 2000; Mújica; Bachmann, 2016). El contenido (sexo, violencia, desastres) y las características estructurales (edición y realización) del tabloide influyen en el arousal emocional (Grabe et al., 2000). La repercusión positiva en el arousal y atención no parecen vinculadas a un impacto significativo con el recuerdo de la información ofrecida (Milburn; McGrail, 1992).

Conocer la respuesta psicológica y fisiológica del público ante los estímulos del storytelling genera hallazgos relevantes para la industria del entretenimiento

\subsection{Contenidos educativos}

Existe una elevada producción académica alrededor del efecto de los programas educativos infantiles sobre la atención. En estos productos la atención está ligada a elementos formales y a la familiaridad con el contenido, entre otros (Barr et al., 2008). La introducción de marionetas, canciones, música animada, efectos de sonido y contenidos secuenciados activan la atención de los niños frente a un decrecimiento ligado a largos segmentos, momentos de baja acción y narración de adultos (Barr et al., 2008). A pesar del obvio interés de la atención en contenidos educativos, por sí sola no es suficiente para motivar el aprendizaje (Bavelier; Green; Dye, 2010). 

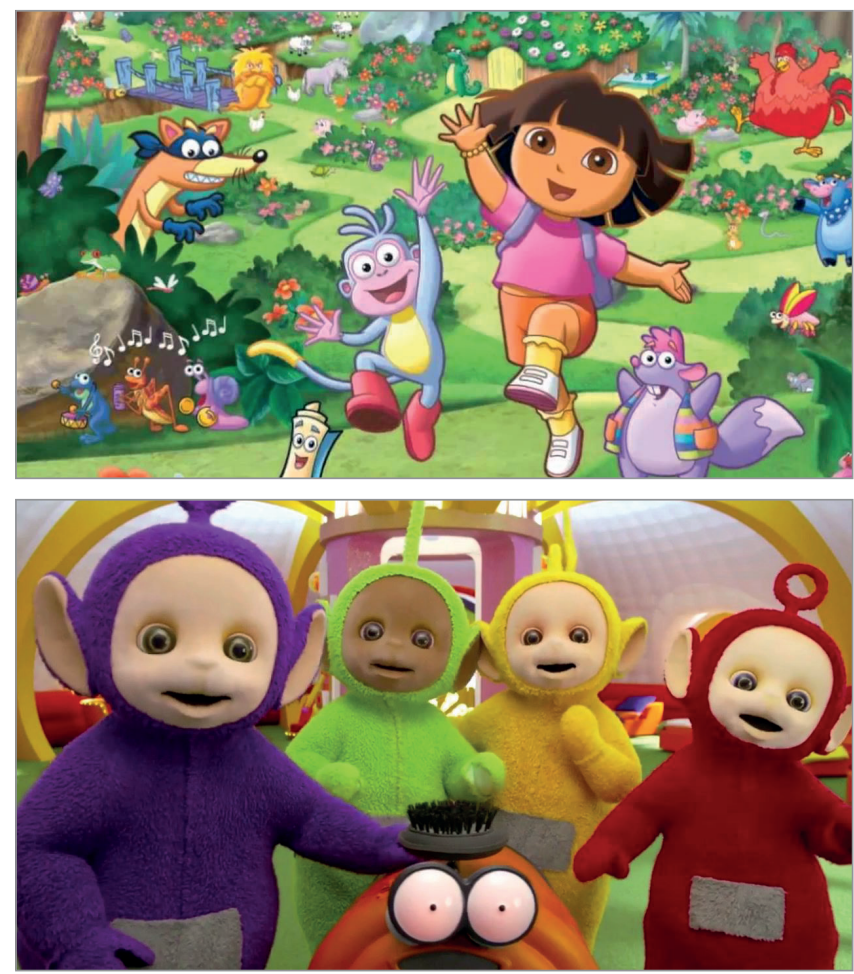

Figura 2. Dora la exploradora y Teletubbies

https://www.youtube.com/watch?v=3fp_H05Q4pw

https://www.youtube.com/watch?v=Fu869sMw9fI

La emoción es clave para aprender y las historias son un gran activador de zonas cerebrales que consolidan el recuerdo (Mora, 2013). En cualquier caso es necesario apuntar que no todas las narrativas influyen por igual en el aprendizaje, aun cuando se trata de contenidos y estructuras aparentemente similares (Bavelier; Green; Dye, 2010). Investigaciones sobre los efectos de diversos programas educativos determinan que mientras programas como 'Dora la exploradora' incrementan las habilidades orales y expresivas en niños de 2 años, el impacto de los 'Teletubbies' sobre el aprendizaje de palabras, atención visual y comprensión es negativo (Linebarger; Walker, 2005). La complejidad de los estímulos visuales y auditivos, sumada a programas con pobres modelos de lenguaje y de participación son inadecuados para el aprendizaje (Linebarger; Walker, 2005).

La eficiencia de los productos educativos infantiles reside en conseguir engagement con la audiencia, introducir personajes que hablen directamente al espectador, promover su participación directa, facilitar oportunidades para responder, introducir una estructura narrativa bien articulada y no sobrecargar con estímulos distractores (Bavelier; Green; Dye, 2010; Linebarger; Walker, 2005). La repetición resulta relevante para incrementar el tiempo de la mirada y facilitar la comprensión (Linebarger; Walker, 2005; Barr et al., 2008). La efectividad también se vincula con contenidos que ayuden a gestionar conflictos sociales, frustración y desacuerdo (Bavelier; Green; Dye, 2010), y con plataformas digitales que favorecen la interactividad, la participación activa y la reiteración de los contenidos, según los neurocientíficos consultados y la bibliografía analizada (Crespo-Pereira; Martínez-Fernández; Campos-Freire, 2017).

Los contenidos audiovisuales contemporáneos han modificado sus pautas estructurales para conseguir mayor eficiencia en términos de atención y emoción

\subsection{Espacio publicitario}

La eficiencia publicitaria es clave para sostener económicamente la industria televisiva, y la neurociencia aporta criterios para repensar el proceso creativo. La mayoría de modelos publicitarios sostienen una relación directa entre la exposición al mensaje y la atención como paso previo para influir emocionalmente e impactar en la memoria y decisión de compra (Woltman-Elpers, 2003). La eficiencia publicitaria se mide mediante el engagement emocional y la atención visual de acuerdo con los expertos y la bibliografía (Warc, 2016; Tapia-Frade; Martín-Guerra; Puente, 2016; Innerscope Research, 2014).

La neurociencia se introduce en la televisión para estudiar la eficacia de los bloques publicitarios e impacto de las nuevas tecnologías en el visionado. Estudios privados buscan conocer cómo activar y mantener la atención durante el bloque comercial como puente para mejorar el recuerdo. El storytelling es preciso para crear fuertes vínculos emocionales con la marca y mejorar su anclaje en la memoria (Micu; Plummer, 2010). El recuerdo a largo plazo se cree básico para predecir el consumo futuro dado que la memoria retiene lo que es más importante para el individuo y revela factores de importancia para dirigir la conducta (Pynta et al., 2014).

Según los expertos consultados, determinar los niveles de atención y emoción de una pieza permitirá a las televisio-

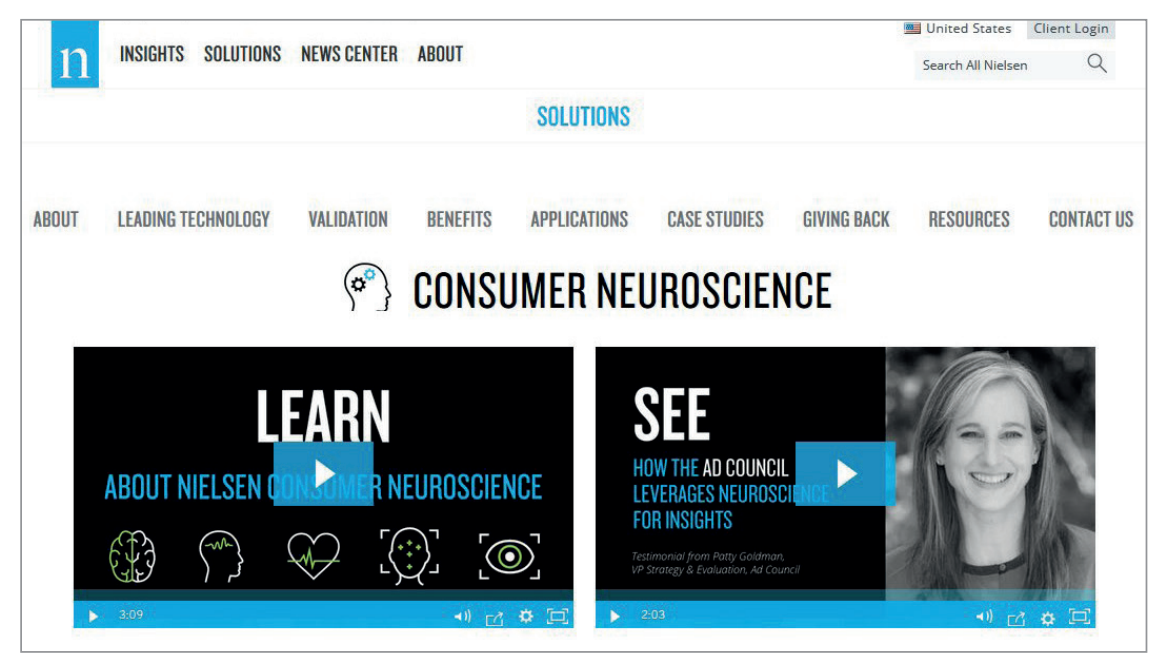

Figura 3. Innerscope Research fue comprada por Nielsen en 2015 http://www.nielsen.com/consumerneuroscience 
nes tomar mejores decisiones y optimizar la inversión de sus anunciantes. Está demostrado que la exposición de mensajes comerciales en un contexto de programación congruente es más efectiva en términos de compresión, atención y carga emocional y codificación de la memoria que los contextos incongruentes (Southwell; Lee, 2004; Brennan, 2011). Grupos como Atresmedia introdujeron en sus bloques publicitarios tres tipos de piezas:

1) 'preguntas-respuestas' o 'datos curiosos' para activar la atención;

2) 'tres anuncios y volvemos' para mantener la atención conseguida durante el programa y favorecer el recuerdo;

3) 'spot antecedente', para fomentar asociaciones entre el spot y una creatividad precedente y vinculada con éste y potenciar el efecto publicitario.

Las televisiones han introducido las técnicas neurocientíficas para optimizar los bloques publicitarios en relación a la atención

\subsection{Nuevas tecnologías}

La neurociencia ha servido para estudiar el impacto del tamaño de la pantalla (Reeves et al., 1999) y la televisión social sobre la atención y emoción. Las plataformas televisivas inmersivas afectan positivamente sobre las conexiones emocionales (Steele et al., 2013). La social TV genera más engagement emocional que la televisión lineal en solitario (Innerscope Research, 2014) mientras que la multipantalla genera altos niveles de engagement y memoria a largo plazo (Pynta et al., 2014; Seixas et al., 2015).

El tamaño de la pantalla determina la respuesta fisiológica del espectador y la eficiencia publicitaria. La pantalla grande registra mayor atención hacia el mensaje frente a la media y pequeña pantalla (Reeves et al., 1999). Aquellos contenidos con violencia y sexo mostraron mayores niveles de arousal en la pantalla grande frente a las pequeñas y medianas (Reeves et al., 1999). Pantallas pequeñas (móvil, tabletas) precisan de diseños de contenido y forma ex profeso para una mejor recepción del mensaje. Las pequeñas pantallas tienen su pico más alto de engagement emocional en los primeros 3-5 segundos del anuncio; por consiguiente, la publicidad debe garantizar ser emocional en este período además de compensar la poca atención visual de la pantalla pequeña incluyendo un copy llamativo (Innerscope Research, 2015).

\section{Conclusiones}

Se observa una tendencia creciente en el empleo de metodología neurocientífica por los medios de comunicación para aportar soluciones científicas objetivas y a medida para la toma de decisiones.

Atención, arousal (emoción) y engagement (compromiso) son las principales variables analizadas sobre contenidos televisivos. Su capacidad para proporcionar análisis segundo a segundo sobre los componentes del storytelling, edición e impacto del soporte, permite identificar cambios potenciales para un mayor rendimiento.

Los estudios de carácter privado se vinculan principalmente al análisis de contenidos de entretenimiento, bloques comerciales y multipantalla y social TV. El diseño a partir de dichos estudios neurocientíficos se postula como un ingrediente más de éxito que acompaña a otras decisiones ejecutivas. La ventaja competitiva asociada a esta metodología determina su opacidad e impide un corpus teórico compartido.

La investigación académica ahonda principalmente en la eficiencia de la historia y la estructura de los contenidos educativos e informativos en términos de atención, emoción y memoria. Se ha detectado una revisión del paradigma en el campo comunicativo, donde la emoción es central para la consecución de los propósitos de contenidos de entretenimiento, especialmente los educativos y publicitarios. Aunque la atención es vital para la secuencia emoción y memoria, el aprendizaje y la actitud vienen determinados principalmente por la emoción y el engagement logrado en la audiencia.

La pequeña pantalla precisa de diseños ex profeso para optimizar la recepción del mensaje

\section{Agradecimientos}

La participación de la primera autora está financiada con cargo a las ayudas de apoyo a la etapa predoctoral del Plan Galego de Investigación, Innovación e Crecemento 20112015 (Plan 12C) de la Consellería de Educación de la Xunta de Galicia (modalidad A-2014). Así mismo, el trabajo forma parte de las actividades de investigación promovidas a través del proyecto del Programa estatal de Fomento de la Investigación Científica y Técnica de Excelencia, subprograma estatal de Generación de Conocimiento del Ministerio de Economía y Competitividad de España sobre "Indicadores de gobernanza, financiación, rendición de cuentas, innovación, calidad y servicio público de las RTV europeas aplicables a España en el contexto digital" (referencia CSO201566543-P), así como de la actividad de la Red Internacional de Investigación de Gestión de la Comunicación (Redes 2016 G-1641 XESCOM) apoyada por la Consellería de Cultura, Educación e Ordenación Universitaria de la Xunta de Galicia (referencia ED341D R2016/019).

\section{Referencias}

Aldama, Frederick (2015). "The science of storytelling. Perspectives from cognitive science, neuroscience, and the humanities". Projections, v. 9, n. 1, pp. 80-95.

https://goo.gl/XnYrHK

https://doi.org/10.3167/proj.2015.090106

Barr, Rachel; Zack, Elisabeth; García, Amaya; Muentener, Paul (2008). "Infants' attention and responsiveness to television increases with prior exposure and parental interaction". Infancy, v. 13, n. 1, pp. 30-56.

https://goo.gl/1Y2GSB

https://doi.org/10.1080/15250000701779378 
Barreda-Ángeles, Miguel (2013). "La investigación sobre la atención durante el consumo de televisión: avances actuales y retos futuros". Historia y comunicación social, v. 18, n. oct., pp. 571-580.

https://doi.org/10.5209/rev_HICS.2013.v18.43989

Bavelier, Daphne; Green, Shawn; Dye, Matthew (2010). "Children, wired: for better and for worse". Neuron, v. 67, n. 9, pp. 692-701.

https://doi.org/10.1016/j.neuron.2010.08.035

Beamish, Gerry; Beamish, Jonathan (2015). "Cave wall to internet, storytelling, the ancient learning art". Industrial and commercial training, v. 47, n. 4, pp. 190-194.

https://doi.org/10.1108/ICT-01-2015-0002

Berliner, Todd (2013). "Hollywood storytelling and aesthetic pleasure". En: Shimamura, Arthur. Psychocinematics: Exploring cognition at the movies. New York: Oxford University Press, pp. 195-212. ISBN: 9780199862139

https://doi.org/10.1093/acprof:oso/9780199862139.003.0010

Bolls, Paul; Lang, Annie; Potter, Robert (2001). "The effects of message valence and listener arousal on attention, memory and facial muscular responses to radio advertisements". Communication research, v. 28, n. 5, pp. 627-651.

https://goo.gl/nr9ZAY

https://doi.org/10.1177/009365001028005003

Brennan, David (2011). "Brainwaves: what neuroscience can teach us about the power of television". En: Television 2011. International key facts.

https://goo.gl/kbLnHx

Cohen, Anna-Lisa; Shavalian, Elliot; Rube, Moshe (2015). "The power of the picture: How narrative film captures attention and disrupts goal pursuit". PLoS one, v. 10, n. 12. https://doi.org/10.1371/journal.pone.0144493

Crespo-Pereira, Verónica; Martínez-Fernández, Valentín-Alejandro; Campos-Freire, Francisco (2017). "Neurociencia para la innovación de contenidos en la televisión pública europea". Comunicar, v. 25, n. 52, pp. 09-18.

https://doi.org/10.3916/C52-2017-01

Crespo-Pereira, Verónica; Martínez-Fernández, Valentín-Alejandro; García-Soidán, Pilar (2016). "El profesional del neuromarketing en el sector audiovisual español". El profesional de la información, v. 25, n. 2, pp. 209-2916.

https://doi.org/10.3145/epi.2016.mar.07

Cutting, James; DeLong, Jordan; Brunick, Kaitlin (2011). "Visual activity in Hollywood film: 1935 to 2005 and beyond". Psychology of aesthetics, creativity, and the arts, v. 5, n. 2, pp. 115-125.

https://goo.gl/qh7XbY

https://doi.org/10.1037/a0020995

Dorr, Michael; Martinetz, Thomas; Gegenfurtner, Karl R.; Barth, Erhardt (2010). "Variability of eye movements when viewing dynamic natural scenes". Journal of vision, v. 10, $n$. 28, pp. 1-17.

https://doi.org/10.1167/10.10.28

Dudai, Yadin (2008). "Enslaving central executives: Toward a brain theory of cinema". Projections, v. 2, n. 2, pp. 21-42.
https://goo.gl/2oWfu4

https://doi.org/10.3167/proj.2008.020203

González-Bernal, Manuel-Ignacio (2016). “Hacia la comprensión del engagement de las audiencias de televisión, modelo conceptual multidimensional desde la comunicación". Palabra clave, v. 19, n. 3, pp. 769-809. https://doi.org/10.5294/pacla.2016.19.3.5

Grabe, Maria-Elizabeth; Zhou, Shuhua; Barnett, Brooke (2001). "Explicating sensationalism in television news: Content and the bells and whistles of form". Journal of broadcasting \& electronic media, v. 45, n. 4, pp. 635-655. https://goo.gl/R77GW8 https://doi.org/10.1207/s15506878jobem4504_6

Grabe, Maria-Elizabeth; Zhou, Shuhua; Lang, Annie; Bolls, Paul-David (2000). "Packaging television news: The effects of tabloid on information processing and evaluative responses". Journal of broadcasting \& electronic media, v. 44, n. 4, pp. 690-703.

https://goo.gl/2kb75N

https://doi.org/10.1207/s15506878jobem4404_4

Hasson, Uri; Landesman, Ohad; Knappmeyer, Barbara; Vallines, Ignacio; Rubin, Nava; Heeger, David (2008). “Neurocinematics: The neuroscience of film". Projections, v. 2, n. 1, pp. 1-26.

https://goo.gl/pDQH3m

https://doi.org/10.3167/proj.2008.020102

Heath, Robert (2007). "Engagement framework". En: Plummer, James; Cook, Bill; Diforio, Don; Schachter, Bert; Sokolyanskaya, Inna; Korde, Tara. Measures of engagement. New York: Advertising Research Foundation, pp. 4-10.

http://gandrllc.com/reprints/Measures_of_Engagement Vol_II_Final_Paper.pdf

Innerscope Research (2014). Welcome to integrated consumer neuroscience on the path to integrated. N.d.: Innerscope. https://goo.gl/5nuzE1

Innerscope Resarch (2015). Innerscope research study shows TV and digital pre-roll significantly stronger platforms for video advertising than Facebook.

https://goo.gl/99NT5z

Jeong, Eui; Bohil, Corey; Biocca, Frank (2011). “Brand logo placements in violent games: Effects of violence cues on memory and attitude through arousal and presence". Journal of advertising, v. 40, n. 3, pp. 59-72.

https://goo.gl/Jr3Ezv

https://doi.org/10.2753/JOA0091-3367400305

Kauttonen, Janne; Kaipainen, Mauri; Tikka, Pia (2014). "Model of narrative nowness for neurocinematic experiments". En: $5^{\text {th }}$ Workshop on computational models of narrative, pp. 77-87.

http://drops.dagstuhl.de/opus/vol/texte/2014/4646/ $p d f / 12 . p d f$

https://doi.org/10.4230/OASIcs.CMN.2014.77

Lang, Annie; Newhagen, John; Reeves, Byron (1996). “Negative video as structure: Emotion, attention, capacity and memory". Journal of broadcasting \& electronic media, v. 40, 
n. 4, pp. $460-477$

https://goo.gl/EBsquk

https://doi.org/10.1080/08838159609364369

Lang, Annie; Potter, Deborah; Grabe, Maria-Elizabeth (2003). "Making news memorable: Applying theory to the production of local television news". Journal of broadcasting \& electronic media, v. 47, n. 1, pp. 113-123.

https://goo.gl/2gA4rr

https://doi.org/10.1207/s15506878jobem4701_7

Lang, Annie; Zhou, Shuhua; Schwartz, Nancy; Bolls, Paul; Potter, Robert (2010). "The effects of edits on arousal, attention and memory for television messages: When an edit can be too much?". Journal of broadcasting \& electronic media, v. 44, n. 1, pp. 94-109.

https://goo.gl/RBGkFS

https://doi.org/10.1207/s15506878jobem4401_7

Linebarger, Deborah; Walker, Dale (2005). “Infants' and toddlers' television viewing and language outcomes". American behavioural scientist, v. 48, n. 5, pp. 624-45.

https://doi.org/10.1177/0002764204271505

Micu, Anca; Plummer, Joseph (2010). "Measurable emotions: how television ads really work". Management slant, v. 50, n. 2.

http://www.deepmetaphors.com/media/MeasEmotions_ JAR_Jun2010.pdf

https://doi.org/10.2501/s002184990909

Milburn, Michael; McGrail, Anne (1992). "The dramatic presentation of news and its effects on cognitive complexity". Political psychology, v. 13, n. 4, pp. 613-632.

https://goo.gl/Kawnko

https://doi.org/10.2307/3791493

Monarth, Harrison (2014). "The irresistible power of storytelling as a strategic business tool". Harvard business review, March 11.

https://hbr.org/2014/03/the-irresistible-power-of-storytellingas-a-strategic-business-tool

Mora, Francisco (2013). Neuroeducación. Madrid: Alianza Editorial. ISBN: 9788420675336

Morin, Christophe (2011). "Neuromarketing: The new science of consumer behavior". Society, v. 48, n. 2, pp. 131-135. https://doi.org/10.1007/s12115-010-9408-1

Mujica, Constanza; Bachmann, Ingrid (2016). "The impact of melodramatic news coverage on information recall and comprehension". Journalism studies, pp. 1-19.

https://doi.org/10.1080/1461670X.2016.1190661

Newhagen, John; Reeves, Byron (1992). "The evening's bad news: Effects of compelling negative television news images on memory". Journal of communication, v. 42, n. 2, pp. 25-41. https://goo.gl/DVXnz4

https://doi.org/10.1111/j.1460-2466.1992.tb00776.x

Pynta, Peter; Seixas, Shaun; Nield, Geoffrey; Hier, James; Millward, Emilia; Silberstein, Richard (2014). "The power of social television: Can social media build viewer engagement?". Advertising research, v. 54, n. 1, pp. 1-15.
https://goo.gl/oN5Jpp

https://doi.org/10.2501/JAR-54-1-071-080

Reeves, Byron; Lang, Annie; Young, Eun; Tatar, Deborah (1999). "The effects of screen size and message content on attention and arousal". Media psychology, v. 1, n. 1, pp. 49-67. https://doi.org/10.1207/s1532785xmep0101_4

Sawyer, Philip (2014). Using neuroscience to test the efficacy of a new advertising format. N.D.: Innerscope Research.

Segev, Idan; Martínez, Luis; Zattorre, Robert (2014). "Brain and art". Frontiers in human neuroscience, v. 8, article 465. https://doi.org/10.3389/fnhum.2014.00465

Seixas, Shaun; Pynta, Peter; Nield, Geoffrey; Silberstein, Robert (2015). "The neuroscience of social television". En: Hajli, Nick. Handbook of research on integrating social media into strategic marketing. Newcastle: IGI Global, pp. 153166. ISBN: 9781466683532

https://doi.org/10.4018/978-1-4666-8353-2.ch010

Sharot, Tali; Phelps, Elizabeth (2004). "How arousal modulates memory: Disentangling the effects of attention and retention". Cognitive, affective \& behavioral neuroscience, $\mathrm{v}$. 4, n. 3, pp. 294-306.

https://goo.gl/kmmYFf

https://doi.org/10.3758/Cabn.4.3.294

Shimamura, Arthur (2010). "Bridging psychological and biological science: The good, bad, and ugly". Perspectives on psychological science, v. 5, n. 6, pp. 772-775.

https://goo.gl/c5rkym

https://doi.org/10.1177/1745691610388781

Shimamura, Arthur (2013). "Psychocinematics: Issues and directions". In: Shimamura, Arthur. Psychocinematics. New York: Oxford University Press, pp. 1-26. ISBN: 978019 9862139

https://doi.org/10.1093/acprof:oso/9780199862139.003.0001

Siefert, Caleb; Gallent, Janet; Jacobs, Devra; Levine, Brian; Stipp, Horst; Marci, Carl (2008). "Biometric and eye-tracking insights into the efficiency of information processing of television advertising during fast-forward viewing". International journal of advertising, v. 27, n. 3, pp. 425-446.

https://doi.org/10.2501/S0265048708080050

Simons, Robert; Detenber, Benjamin; Cuthbert, Bruce; Schawartz, David; Reiss, Jason (2003). "Attention to television: Alpha power and its relationships to image motion and emotional content". Media psychology, v. 5, n. 3, pp. 283-301. https://goo.gl/ozabAF

https://doi.org/10.1207/S1532785XMEP0503_03

Smith, Tim (2013). "Watching you watch movies: using eye tracking to inform cognitive film theory". In: Shimamura, Arthur. Psychocinematics. New York: Oxford University Press, pp. 165-191. ISBN: 9780199862139 https://doi.org/10.1093/acprof:oso/9780199862139.003.0009

Southwell, Brian; Lee, Mira (2004). "A pitfall of new media? User controls exacerbate editing effects on memory". Journalism \& mass communication quarterly, v. 81, n. 3, pp. 643-656. 
https://doi.org/10.1177/107769900408100311

Steele, Audrey; Jacobs, Devra; Siefert, Caleb; Rule, Randall; Levine, Brian; Marci, Carl (2013). "Leveraging synergy and emotion in a multi-platform world". Journal of advertising research, pp. 417-430.

https://doi.org/10.2501/JAR-53-4-417-430

Tapia-Frade, Alejandro; Martín-Guerra, Elena (2016a). “El estilo cómico en publicidad. Un análisis de su eficiencia desde la neurociencia". Comunicación y sociedad, n. 27, pp. 121-144. https://goo.gl/WiXxrE

Tapia-Frade, Alejandro; Martín-Guerra, Elena (2016b). "Neurociencia aplicada a la televisión: medición de la atención y la emoción de la serie 'Forever'”. Vivat academia, n. 13, pp. 69-82.

https://doi.org/10.15178/va.2016.134.69-82

Tapia-Frade, Alejandro; Martín-Guerra, Elena; Puente, José-Enrique (2016). "Neurociencia y publicidad. Atención, emoción y su relación con los premios obtenidos en el Festival internacional de publicidad de Cannes". Anàlisi. Quaderns de comunicació i cultura, v. 54, pp. 75-95.

https://ddd.uab.cat/record/166319

https://doi.org/10.7238/a.v0i54.2613

Thorson, Esther (2011). "Using eyes on screen as a measure of attention to television". En: Lang, Annie. Measuring psychological responses to media messages. New Jersey: Routledge, pp. 65-84. ISBN: 9780415515566
Thorson, Esther; Lang, Annie (1992). "The effects of television videographics and lecture familiarity on adult cardiac orienting responses and memory". Communication research, v. 19, n. 3, pp. 346-369.

https://goo.gl/yLXARP

https://doi.org/10.1177/009365092019003003

Warc (2016). "Turner scores with neuroscience". Warc, April 4.

https://goo.gl/eeeENq

Welbourne, Theresa (2015). "Data-driven storytelling: The missing link in HR data analytics". Employment relations today, pp. 27-33.

https://goo.gl/WhDcR7

https://doi.org/10.1002/ert.21471

Woltman-Elpers, Josephine (2003). Consumers' moment-to-moment processing of television commercials. Groningen: s.n. ISBN: 9053350209

http://www.rug.nl/research/portal/files/3002327/thesis.pdf

Zak, Paul (2014). "Why your brain loves good storytelling". Harvard business review, October 28.

https://hbr.org/2014/10/why-your-brain-loves-goodstorytelling

Zhou, Shuhua (2005). "Effects of arousing visuals and redundancy on cognitive assessment of television news". Journal of broadcasting \& electronic media, v. 49, n. 1, pp. 23-42. https://doi.org/10.1207/s15506878jobem4901_3

\section{Inforảrea}

\section{Ayudamos a tu organización en la transformación digital y el gobierno de la información}

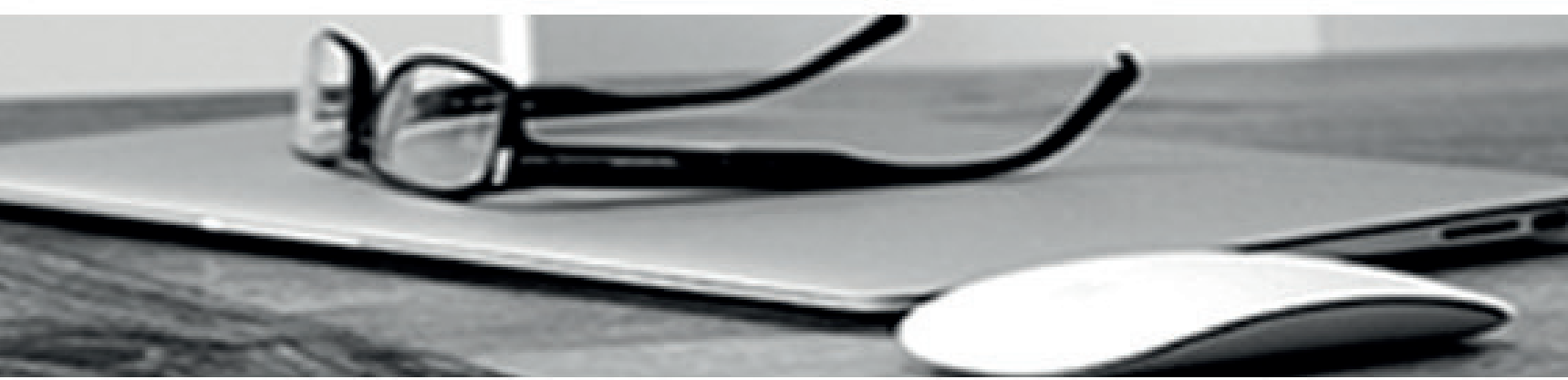

* Consultoría estratégica en gestión y gobierno de la información

* "Gestión documental y "records management"

* Gestión de contenidos, intranets corporativas y entornos de colaboración

* Estudios especializados

Clientes satisfechos, cientos de empresas nacionales e internacionales y más de 30 años de experiencia son la mejor garantía de nuestra reputación. 